\title{
HeartMate 3: Facing the challenge of past success
}

\author{
William E. Stansfield, MD, and Vivek Rao, MD, PhD
}

\author{
From the University of Toronto, Toronto General Hospital, Toronto, Ontario, Canada. \\ Received for publication Jan 26, 2016; revisions received April 6, 2016; accepted for publication April 15, 2016; \\ available ahead of print May 20, 2016. \\ Address for reprints: William E. Stansfield, MD, Department of Surgery, University of Toronto, 4N-466 Toronto \\ General Hospital, 200 Elizabeth St, Toronto, Ontario, Canada M5G2C4 (E-mail: william.stansfield@uhn.ca). \\ J Thorac Cardiovasc Surg 2016;152:683-5 \\ $0022-5223 / \$ 36.00$ \\ Copyright $(2016$ by The American Association for Thoracic Surgery \\ http://dx.doi.org/10.1016/j.jtcvs.2016.04.048
}

2001 was an exciting year. Apple released the first iPod and the Blackberry was catching on as a new way to do e-mail. For heart failure clinicians, the Randomized Evaluation of Mechanical Assistance for the Treatment of Congestive Heart Failure (REMATCH) trial $^{1}$ conclusively demonstrated the superiority of the HeartMate XVE LVAD over optimum medical management for patients with end-stage heart failure who were not eligible for transplant. The legacy of this trial is that new devices for advanced heart failure can no longer be evaluated against medical therapy. Instead, they must be evaluated against the current best devices. The newest entry, the HeartMate 3 (HM3), aims to improve on existing devices with 3 key features: (1) an artificial pulse, (2) full magnetic levitation, and (3) textured interior surfaces.

The pulsatile-flow HeartMate XVE was phased out of clinical use in $2009-2010^{2}$ and was replaced by the continuous axial flow HeartMate II. Smaller, lighter, and much more durable, this pump was rapidly adopted and implant rates soared. ${ }^{3}$ The HeartWare HVAD joined the market only a few years later with a continuous centrifugal-flow design. Comparisons between the new continuous-flow pumps and the XVE pulsatile pump identified several complications specific to continuousflow technology, including aortic insufficiency (AI) ${ }^{4}$ and gastrointestinal (GI) bleeding. ${ }^{5}$ GI bleeding is a complex, multifactorial issue, but loss of pulsatility contributes to the development of arteriovenous malformations that are associated with GI bleeding events. ${ }^{6}$ Continuous pressurization of the aortic root likely contributes to the development of AI. ${ }^{7}$ The HM3 was designed to address these issues by adding intermittent speed reduction to a continuous centrifugal-flow design.

Pulsatility with continuous-flow devices is not an all-or-none phenomenon. Most patients with continuousflow LVADs have some degree of arterial pulsatility, leading many to discourage the term "non-pulsatile.," Pulsatility may result from blood ejected through the aortic valve or transmission of the systolic pressure wave through the LVAD (in cases of a closed aortic

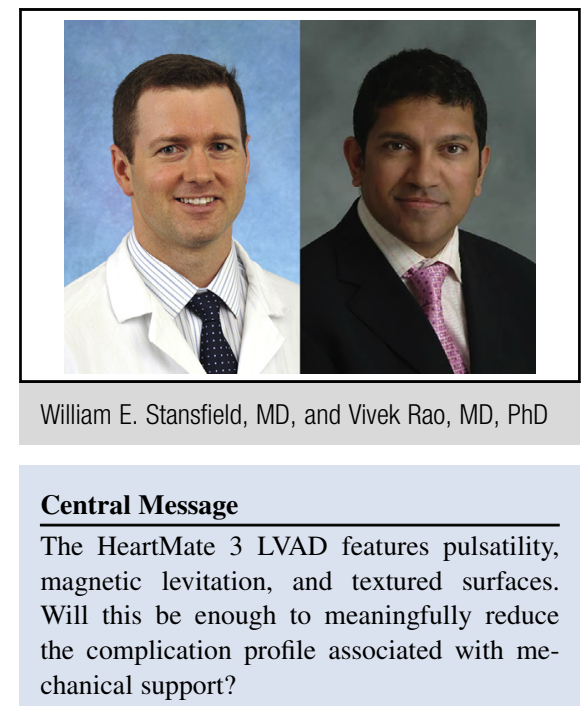

\section{Perspective}

Continuous-flow left ventricular assist devices (LVADs) revolutionized mechanical support but decreased pulsatility, and the tradeoff between bleeding and thrombosis continues to limit more widespread adoption. The HeartMate 3 LVAD adds an artificial pulse to an established continuous-flow design, and has additional features for improved biocompatibility. Results from the CE Mark trial are promising. Is this new pump ready for prime time?

See Editorial Commentary page 686.

valve). The HeartMate II and HVAD both identify changes in power (and corresponding flow) during systole and diastole using either a pulsatility index or a flow waveform. These measures reflect ventricular contractility and correlate with the pulse pressure on arterial pressure tracings. More recent reports indicate that the pulsatility index is inversely related to GI bleeding, ie, patients with higher pulsatility demonstrate fewer incidences of GI bleeding. ${ }^{9-11}$ Because the artificial pulse in the HM3 is not synchronized with patient heart rate, it may augment or diminish the native pulse. Should clinicians therefore reserve the artificial pulse for those patients with poor pulsatility?

The development of AI while on mechanical support can be debilitating and life threatening. The result is a decrease in net forward flow, increased left ventricular distension, and a return to heart failure. One strategy to 


$$
\begin{aligned}
& \text { Abbreviations and Acronyms } \\
& \begin{aligned}
\text { AI } & =\text { aortic insufficiency } \\
\text { BTT } & =\text { bridge-to-transplant } \\
\text { GI } & =\text { gastrointestinal } \\
\text { HM3 } & =\text { HeartMate } 3 \\
\text { LVAD } & =\text { left ventricular assist device }
\end{aligned}
\end{aligned}
$$

stave off this complication has been to adjust LVAD speed (and corresponding flows) down in order to better fill the ventricle and enable ejection through the aortic valve. This is a narrow line to walk, however, because it risks a return to a low-cardiac-output state if the patient's ventricle cannot keep up with the added workload. The ideal pump would enable adequate decompression of the left ventricle while still allowing enough pressure variation at the aortic valve to generate intermittent aortic valve opening, and thus prevent development of AI. The pulse wave from the HM3 is small compared with a pulsatile-flow LVAD or the pulse of a normal ventricle but is quite significant compared with existing continuous-flow devices. Depending on loading conditions, LVAD speed, and intrinsic contractility, this pulse may be greater in magnitude than that of a patient's own ventricle. Many clinicians are hopeful that this small pulse will help prevent AI, but we will have to wait for more clinical data to know for sure.

More recent studies have identified lower blood pressure as protective against the development of $\mathrm{AI} .{ }^{12}$ In addition, lower blood pressure is increasingly attractive as a therapeutic target because of newly described associations with lower rates of hemorrhagic and embolic stroke. ${ }^{13,14}$ If clinicians focus more effort on blood pressure control, this may also improve pulsatility, and thus help prevent both GI bleeding and development of AI. Will the artificial pulse be helpful in all LVAD patients, or can we identify a subgroup of patients who are most likely to benefit? Duration of support has also been related to development of AI, so it will likely take some time to answer these types of questions. ${ }^{12}$

Current practice guidelines for implantable LVADs in North America involve both an antiplatelet agent and anticoagulation with a vitamin $\mathrm{K}$ antagonist. Despite the acquired von Willebrand factor deficiency associated with most continuous-flow LVADs, ${ }^{15}$ pump thrombosis ${ }^{16}$ and the risk of thromboembolic events are major concerns. Balancing the risk of bleeding and the risk of thrombosis for a given patient can prove challenging.

The design goal for the HM3 was a level of biocompatibility that would only require daily aspirin and no other antithrombotic medication. ${ }^{17}$ Full magnetic levitation is hypothesized to help prevent thrombosis by allowing larger flow path gaps around the rotor, and by eliminating the need for a mechanical or hydrodynamic bearing. Texturing of the pump lining with sintered titanium microspheres may also promote the formation of a biologic barrier to further help decrease the risk of thrombosis. $^{18,19}$ Despite these advanced features, antithrombosis guidelines for the HM3 CE Mark trial were nearly the same as those in the HeartMate II bridge-to-transplant (BTT) US trial: postoperative heparin until therapeutic international normalized ratio, and daily aspirin $(81 \mathrm{mg})$. Looking at the rates of bleeding in the 2 trials (outside the perioperative period), HM3 compares favorably with HeartMate II; $22 \%$ of patients experienced significant bleeding with the HM3 over 5 months (including GI bleeding), and $23 \%$ of patients had bleeding in the HeartMate II trial over 17 months. ${ }^{20,21}$ The crucial question is whether or not the new design features of the HM3 will allow clinicians to reduce antithrombotic therapy to levels that significantly reduce bleeding episodes?

Stopping anticoagulation altogether is not a new idea in the mechanical circulatory support community. Recurrent bleeding has led many clinicians to stop aspirin and warfarin in certain patients, ultimately leading to the STudy of Reduced Anti-Coagulation/Anti-platelEt Therapy in Patients with the HeartMate II LVAS (TRACE). In TRACE, 100 HeartMate II patients with recurrent bleeding were enrolled and followed for 1 year with reduced antithrombotic medications, ie, no aspirin, no warfarin, or no medications. ${ }^{22}$ In these patients, the incidence of device thrombosis was uncommon at $4 \%$, but $40 \%$ of patients off all antithrombotic medications continued to have bleeding complications. Clearly then, one size of anticoagulation does not fit all patients. Efforts to tailor antithrombotic medications more precisely have led to a wide range of studies of platelet function, coagulation, and hemolysis in patients with an LVAD. These studies identify large differences between patients in their responsiveness to antiplatelet therapies, and in the effect of a continuous-flow LVAD on platelet aggregation, lactate dehydrogenase, and D-dimers. ${ }^{23}$ If the HM3 does not allow reduction in antithrombotic treatments for all patients, then we will need a better understanding of how LVADs affect the thrombosis system in order to safely individualize antiplatelet and anticoagulation therapies.

In REMATCH, 68 patients were treated with an LVAD. In the HeartMate II BTT trial, 281 patients received an LVAD. MOMENTUM 3, the US trial for the HM3, will enroll 1000 patients. With each new device, the bar is set progressively higher, and the effort to reach that bar 
increases proportionately. Dramatic advances have been made in the standard of care for patients with end-stage heart failure in the last 15 years. With increasing experience, understanding, and technology, what will we achieve in the next 15 years?

\section{Conflict of Interest Statement}

Dr Rao is a consultant for both Thoratec (St. Jude) and HeartWare. The other author has nothing to disclose with regard to commercial support.

\section{References}

1. Rose E, Gelijns A, Moskowitz A, Heitjan D, Stevenson L, Dembitsky W, et al. Long-term use of a left ventricular assist device for end-stage heart failure. NEJMed. 2001:345:1435-43.

2. Kirklin JK, Naftel DC, Kormos RL, Stevenson LW, Pagani FD, Miller MA, et al. The Fourth INTERMACS Annual Report: 4,000 implants and counting. J Heart Lung Transplant. 2012;31:117-26.

3. Kirklin JK, Naftel DC, Pagani FD, Kormos RL, Stevenson LW, Blume ED, et al. Seventh INTERMACS annual report: 15,000 patients and counting. $J$ Heart Lung Transplant. 2015;34:1495-504.

4. Cowger J, Pagani FD, Haft JW, Romano MA, Aaronson KD, Kolias TJ. The development of aortic insufficiency in left ventricular assist device-supported patients. Circ Heart Fail. 2010;3:668-74.

5. Crow S, John R, Boyle A, Shumway S, Liao K, Colvin-Adams M, et al. Gastrointestinal bleeding rates in recipients of nonpulsatile and pulsatile left ventricular assist devices. J Thorac Cardiovasc Surg. 2009;137:208-15.

6. Demirozu ZT, Radovancevic R, Hochman LF, Gregoric ID, Letsou GV, Kar B, et al. Arteriovenous malformation and gastrointestinal bleeding in patients with the HeartMate II left ventricular assist device. J Heart Lung Transplant. 2011;30:849-53.

7. Cowger J, Rao V, Massey T, Sun B, May-Newman K, Jorde U, et al. Comprehensive review and suggested strategies for the detection and management of aortic insufficiency in patients with a continuous-flow left ventricular assist device. J Heart Lung Transplant. 2015;34:149-57.

8. Slaughter MS. Long-term continuous flow left ventricular assist device support and end-organ function: prospects for destination therapy. J Card Surg. 2010; 25:490-4.

9. Wever-Pinzon O, Selzman CH, Drakos SG, Saidi A, Stoddard GJ, Gilbert EM, et al. Pulsatility and the risk of nonsurgical bleeding in patients supported with the continuous-flow left ventricular assist device HeartMate II. Circ Heart Fail. 2013;6:517-26.

10. Grosman-Rimon L, Tumiati LC, Fuks A, Jacobs I, Lalonde SD, Cherney DZ, et al. Increased cyclic guanosine monophosphate levels and continuous-flow left-ventricular assist devices: Implications for gastrointestinal bleeding. J Thorac Cardiovasc Surg. 2016;151:219-27.
11. Jabbar HR, Abbas A, Ahmed M, Klodell CT Jr, Chang M, Dai Y, et al. The incidence, predictors and outcomes of gastrointestinal bleeding in patients with left ventricular assist device (LVAD). Dig Dis Sci. 2015;60:3697-706.

12. Patil NP, Mohite PN, Sabashnikov A, Dhar D, Weymann A, Zeriouh M, et al Does postoperative blood pressure influence development of aortic regurgitation following continuous-flow left ventricular assist device implantation? Eur J Cardiothorac Surg. 2016;49:788-94.

13. Lampert BC, Eckert C, Weaver S, Scanlon A, Lockard K, Allen C, et al. Blood pressure control in continuous flow left ventricular assist devices: efficacy and impact on adverse events. Ann Thorac Surg. 2014;97:139-46.

14. Nassif ME, Tibrewala A, Raymer DS, Andruska A, Novak E, Vader JM et al. Systolic blood pressure on discharge after left ventricular assist device insertion is associated with subsequent stroke. J Heart Lung Transplant 2015;34:503-8.

15. Crow S, Milano C, Joyce L, Chen D, Arepally G, Bowles D, et al. Comparative analysis of von Willebrand factor profiles in pulsatile and continuous left ventricular assist device recipients. ASAIO J. 2010;56:441-5.

16. Starling RC, Moazami N, Silvestry SC, Ewald G, Rogers JG, Milano CA, et al. Unexpected abrupt increase in left ventricular assist device thrombosis. $N$ Engl J Med. 2014;370:33-40.

17. Farrar DJ, Bourque K, Dague CP, Cotter CJ, Poirier VL. Design features, developmental status, and experimental results with the Heartmate III centrifugal left ventricular assist system with a magnetically levitated rotor. ASAIO J. 2007;53: 310-5.

18. Schmitto JD, Hanke JS, Rojas SV, Avsar M, Haverich A. First implantation in man of a new magnetically levitated left ventricular assist device (HeartMate III). J Heart Lung Transplant. 2015;34:858-60.

19. Rose EA, Levin HR, Oz MC, Frazier OH, Macmanus Q, Burton NA, et al. Artificial circulatory support with textured interior surfaces. A counterintuitive approach to minimizing thromboembolism. Circulation. 1994;90:II87-91.

20. Netuka I, Sood P, Pya Y, Zimpfer D, Krabatsch T, Garbade J, et al. Fully magnet ically levitated left ventricular assist system for treating advanced HF: a multicenter study. J Am Coll Cardiol. 2015;66:2579-89.

21. Pagani FD, Miller LW, Russell SD, Aaronson KD, John R, Boyle AJ, et al. Extended mechanical circulatory support with a continuous-flow rotary left ventricular assist device. J Am Coll Cardiol. 2009;54:312-21.

22. Katz JN, Adamson RM, John R, Tatooles A, Sundareswaran K, Kallel F, et al Safety of reduced anti-thrombotic strategies in HeartMate II patients: a oneyear analysis of the US-TRACE Study. J Heart Lung Transplant. 2015;34: 1542-8.

23. Birschmann I, Dittrich M, Eller T, Wiegmann B, Reininger AJ, Budde U, et al Ambient hemolysis and activation of coagulation is different between HeartMate II and HeartWare left ventricular assist devices. J Heart Lung Transplant. 2014 33:80-7.

Key Words: MCS, mechanical circulatory support, heart failure, HeartMate 3, LVAD, left ventricular assist device 